Revue européenne des sciences sociales

European Journal of Social Sciences

XLVI-141 | 2008

Processus d'évaluation des sciences sociales: acteurs et valeurs

\title{
Évaluation, mesure, mouvement : la philosophie contre la science et les concepts du cinéma (Bergson, Marey)
}

\section{Maria Tortajada}

\section{OpenEdition}

\section{Journals}

Édition électronique

URL : http://journals.openedition.org/ress/155

DOI : $10.4000 /$ ress. 155

ISSN : $1663-4446$

\section{Éditeur}

Librairie Droz

\section{Édition imprimée}

Date de publication : 1 mai 2008

Pagination : 95-111

ISBN : 978-2-600-01220-1

ISSN : 0048-8046

\section{Référence électronique}

Maria Tortajada, «Évaluation, mesure, mouvement : la philosophie contre la science et les concepts du cinéma (Bergson, Marey) ", Revue européenne des sciences sociales [En ligne], XLVI-141 | 2008, mis en ligne le 01 mai 2011, consulté le 19 avril 2019. URL : http://journals.openedition.org/ress/155 ; DOI : 10.4000/ress. 155 
Maria TORTAJADA

\section{ÉVALUATION, MESURE, MOUVEMENT: LA PHILOSOPHIE CONTRE LA SCIENCE ET LES CONCEPTS DU CINÉMA (BERGSON, MAREY)}

Lorsqu'on évalue, on porte un jugement sur un objet, un fait, un acte, un phénomène, un travail, une recherche, un projet dont on tente de cerner la valeur. Celle-ci est déterminée par le contexte idéologique ou expérimental dans lequel on l'élabore ou la produit comme telle, qu'elle soit qualitative ou quantitative, qu'il s'agisse de valeur ou d'intérêt - dans le sens de Pierre Livet. Cependant, outre les critères de qualité ou de quantité dans la définition de la valeur, l'évaluation suppose une étape incontournable qui consiste en la mise en discours de l'objet évalué. Pour dire la valeur de quelque chose, il faut formuler sa position tout en faisant référence à l'objet évalué, qui peut être lui-même de nature discursive, mais qui n'échappe pas à une description ou redescription par l'évaluateur lui-même. La manière dont ce dernier présente l'objet soumis à son analyse impose sa reconstruction et sa remodélisation en fonction de la démonstration visée, c'est-à-dire la valorisation ou dévalorisation engagée par le discours. Mais cette reformulation n'est pas toujours d'emblée repérable. Tel discours peut reprendre les termes exacts qualifiant un objet, une recherche, un projet, une expérience, tout en modifiant d'un point de vue épistémologique les concepts qui y sont mis en jeu.

Ce phénomène déborde le contexte très concret qui touche aux divers processus d'évaluation de la recherche aujourd'hui. Il est impliqué dans tout débat intellectuel ou scientifique. Sa généralité n'en fait pas pour autant un phénomène secondaire dans l'évaluation. Il est au cœur de l'argumentation, de l'effet de «preuve» dans le processus d'évaluation. Nous le traiterons à travers un exemple concret qui fait partie aujourd'hui de l'histoire. Dans la mesure où nous viserons à dégager un mécanisme d'appropriation conceptuelle, nous espérons que cette réflexion pourra être rapportée à une interrogation plus large sur l'évaluation en sciences humaines.

L'objet qui nous servira d'exemple est particulièrement intéressant pour la réflexion sur l'évaluation car il propose de revenir sur un moment fort du débat opposant la valeur qualitative à la quantification qu'opère la science. La critique développée par Henri Bergson fait date dans la mise en cause des pratiques de quantification indispensables à l'expérimentation scientifique. La question de la mesure sera donc traitée dans les lignes qui suivent comme l'objet de l'évaluation bergsonienne et non pas comme un critère d'évaluation à interroger en tant que tel. Cependant, la notion de mesure sera essentielle, car elle participe à construire les concepts et les relations qui les unissent à l'intérieur des discours que nous allons analyser. 
Nous mènerons cette réflexion dans le champ de compétence qui est le nôtre. Dans une perspective de renouvellement de l'histoire du cinéma, nous développons l'étude épistémologique des dispositifs de vision et d'audition ${ }^{1}$. Il s'agit de cerner une ou plusieurs définitions du «cinéma» en relation avec d'autres machines ou d'autres dispositifs qui lui sont parents, en observant les mutations conceptuelles à l'œuvre à telle ou telle époque ${ }^{2}$. Il n'y a pas un seul dispositif historique du cinéma - qui serait celui de la salle obscure par exemple - mais plusieurs. Il n'y a pas seulement des «actualisations» historiques des dispositifs, mais aussi bien des occurrences proprement discursives. Etudier le statut du cinéma autour de 1900, c'est dégager la ou les «idées du cinéma» telles qu'elles s'élaborent à cette époque, non pas seulement dans les pratiques spectaculaires ou documentaires mais aussi dans les discours. Il faut pour cela mettre en évidence les notions et les concepts associés à tel dispositif ainsi que les relations qui les font travailler ensemble, ce qui suppose de cerner la fonction théorique du dispositif à l'intérieur d'une discipline qui se l'approprie, d'un discours spécifique qui l'utilise et le fait fonctionner dans sa propre logique: ainsi de Bergson avec le cinématographe. Nous allons aborder ici la question de l'évaluation en rapport avec une interrogation sur le statut du «cinéma» autour de 1900, c'est-à-dire sur les concepts du «cinéma» ${ }^{3}$ au moment de son émergence.

\section{BERGSON ET LA SCIENCE : LA CHRONOPHOTOGRAPHIE}

Henri Bergson propose une évaluation de la science antique et moderne pour en faire la critique à l'aune de l'intuition: en voulant mesurer le mouvement, qui est le principe même de la vie, la science ne cesse de le décomposer. Pour mieux le connaître, elle le trahit, et en définitive l'arrête. Elle ne traite que d'un temps

\footnotetext{
1 Le terme de dispositif est fréquent aujourd'hui dans différents champs du savoir, en histoire du cinéma, en histoire de l'art, en théorie littéraire, en philosophie (notamment Agambem, 2007; Vouilloux, 2007). Nous le définirons ici de manière pragmatique. Par dispositif, il faut entendre le système de rapports qui s'instaure entre le spectateur et la représentation, tenant compte du mode de production et de monstration de cette représentation. La projection, par exemple, est un élément essentiel du dispositif cinématographique le plus connu au $\mathrm{XX}^{\mathrm{e}}$ siècle, ainsi que le défilement photogrammatique.

2 La Section d'histoire et esthétique du cinéma de l'Université de Lausanne développe des recherches autour du projet «Epistémè 1900 » entendu comme un préalable pour comprendre les mutations épistémologiques à l'œuvre aujourd'hui, notamment autour des nouvelles technologies (Albera, Tortajada, 2004; Tortajada, 2004).

3 Notre définition s'inspire du concept scientifique chez Gaston Bachelard. Selon ce dernier, il faut mettre à distance la signification pour dégager l' explication épistémologique d'un terme, qui n'est plus alors la dénomination d'une notion, mais le fait inséré dans son contexte de production et d'expérimentation: «A une même époque, sous un même mot, il y a des concepts si différents! Ce qui nous trompe, c'est que le même mot à la fois désigne et explique. La désignation est la même; l'explication est différente» (Bachelard, Milna/Paris, Mazzotta/La Cinémathèque française, 1999 (1938), p.17). Cette approche se distingue radicalement de la démarche deleuzienne (Deleuze, 1983 et 1985) qui cherche à créer dans la philosophie les concepts du cinéma. Voir sur ce point l'analyse de Dominique Château (2003, pp. 104-110).
} 
homogène, qui en fait n'existe pas vraiment. Le modèle négatif de cette analyse est le cinématographe, connu en 1907 pour les spectacles qu'il permet de produire.

Bergson a joué un rôle important dans la théorisation esthétique au $\mathrm{XX}^{\mathrm{e}}$ siècle en art, mais aussi pour le cinéma. Sa référence au cinématographe en est certainement en grande partie responsable. Dans L'évolution créatrice (1907), il fait de cette machine le modèle de la pensée, de la connaissance scientifique, de la perception et du langage après avoir élu la photographie comme métaphore de la perception dans Matière et mémoire (1896) ${ }^{4}$. Gilles Deleuze s'est particulièrement intéressé à la part du cinéma dans la philosophie de Bergson pour montrer la dimension cinématographique de ses thèses sur le mouvement. Il retourne pour cela Bergson contre lui-même, car le rôle éminent accordé dans L'évolution créatrice au cinématographe reste négatif, renvoyant non pas à l'intuition bergsonienne ou à ce qui s'y rapporte, mais à ce qui la nie. C'est bien dans l'emploi qu'en fait Bergson qu'il nous paraît essentiel de comprendre le «cinéma».

La référence de Bergson au cinématographe pourrait surprendre de prime abord: comment un discours philosophique peut-il choisir un appareil destiné aux spectacles populaires qui se donnent dans le foires pour expliquer rien moins que le fonctionnement de la science? Il faut situer ce dispositif dans son contexte pour saisir le geste bergsonien. Le cinématographe de Bergson appartient à l'espace de la chronophotographie, et celle-ci s'élabore dans le champ de la science.

Lorsqu'il fait du cinématographe le modèle de la pensée scientifique, Bergson est homme de son temps: celui qui voit l'émergence du cinéma certes, mais aussi celui qui se passionne pour toutes les questions du mouvement. Ce phénomène, si essentiel au bergsonisme, est ce qui attire les foules vers le cinématographe, cette machine capable de restituer leur dynamique aux images des êtres et des choses. Mais le mouvement - que durant le XIX ${ }^{\mathrm{e}}$ siècle on s'amuse à composer et décomposer grâce à des objets de salon ou de spectacle comme le phénakistiscope, le praxinoscope, ou d'autres ingénieux systèmes fondés sur la persistance rétinienne - le mouvement est d'abord objet de la science. De l'invention de Plateau jusqu'à la chronophotographie, lieu essentiel de théorisation et d'expérimentation, le mouvement est au cœur des réflexions.

Les travaux d'Etienne-Jules Marey sont de ce point de vue remarquables: développant en physiologiste ses études sur le mouvement, Marey invente des instruments capables de le décomposer et de le synthétiser. Dans la pratique chronophotographique se finalisent un certain nombre d'inventions et d'améliorations techniques qui permettront les projections cinématographiques. Dans l'histoire traditionnelle du cinéma, Marey rivalise même avec Lumière pour le titre «d'inventeur ». Certes, d'autres pratiquent l'enregistrement du mouvement grâce à une technique photographique et procèdent à sa synthèse, avec des finalités diverses, parmi lesquelles le spectacle de l'image animée: ainsi de Muybridge, Anschütz ou

4 Le chapitre IV de L'évolution créatrice s'intitule «Le mécanisme cinématographique de la pensée et l'illusion mécanistique». Bergson est souvent cité ou commenté dans les débats sur la question du cinéma comme art autour des années 10, et il sera textuellement repris par Jean Epstein dans les années 40 (Epstein, 1946), par André Bazin dans les années 50 (Bazin, 1985 (1975)), et Gilles Deleuze $(1983,1985)$ dans les années 80. 
Edison. Mais Marey ne joue pas seulement un rôle essentiel pour ses inventions. A travers ses grandes synthèses sur la chronophotographie ${ }^{5}$, il fait non seulement œuvre de scientifique mais aussi d'historien, passant en revue les travaux des autres pionniers. Il dessine les contours de la chronophotographie dans son contexte d'expérimentation. Il en construit la dimension théorique et symbolique, associée à une méthode scientifique. Sa voix porte d'autant plus que, autour de 1900, Marey occupe une place institutionnelle forte et visible ${ }^{6}$. En somme, à travers son discours théorique, Marey élabore les concepts de la chronophotographie, qui sont ceux d'une certaine idée du «cinéma»: nous retiendrons le mouvement, l'instant, le photogramme, l'intervalle et la mesure. La notion de mesure semble très secondaire dans le dispositif cinématographique tel qu'il s'est généralisé au $\mathrm{XX}^{\mathrm{e}}$ siècle avec les projections dans la salle obscure. Mais au moment Marey, lorsque se jouent les avancées techniques et conceptuelles dont bénéficiera le cinéma, les concepts du «cinéma» sont inséparables de la mesure.

Si l'on peut dire que le cinématographe de Bergson s'inscrit dans l'espace de la chronophotographie, c'est certainement parce qu'on retrouve, dans différents ouvrages de Bergson, les traces d'exemples empruntés au débat sur la chronophotographie, autour d'images qui ont marqué l'époque: l'homme qui court ou le galop du cheval'. C'est aussi parce que, dans le contexte de l'époque, le Cinématographe Lumière est un chronophotographe: c'est ainsi que le présente Marey à l'exposition universelle de $1900^{8}$. Mais le lien du cinématographe bergsonien et de la chronophotographie apparaît surtout dans la fonction que le philosophe lui accorde en faisant de lui le modèle de la pensée scientifique. Si le cinématographe convient parfaitement à la visée de Bergson, c'est parce qu'il s'inscrit dans un contexte où le «cinéma » est pensé à partir d'une pratique scientifique qui cherche à décomposer le mouvement - ce que Bergson critiquera -, d'une pratique qui impose ses concepts : la notion de mesure y est déterminante ${ }^{9}$. En somme, on peut dire que les concepts de la science tels qu'ils sont modélisés par Bergson dans le cinématographe prennent pour référence ceux du «cinéma» dans son état chronophotographique.

Il ne faut pas s'arrêter là. La compréhension des notions de mouvement et de mesure avancées par Bergson implique de saisir l'utilisation implicite que fait le philosophe de Marey dans son évaluation de la démarche scientifique. Cette utilisation n'est pas neutre; elle impose une transformation. Ce qui nous intéresse est que, dans l'évaluation opérée par Bergson, il y a reformulation des concepts mareysiens, et que, par conséquent, les notions liées à l'idée même de «cinéma»

5 Marey, 1885, 1890, 1894, 1900.

6 Marey n'est pas seulement le scientifique réputé, reconnu par ses pairs, professeur au Collège de France. Ses interventions visent à diffuser sa méthode et ses inventions, comme les publications dans La Nature ou son exposition d'instruments à l'Exposition universelle internationale de 1900.

7 Voir sur ce point Anson Rabinbach (2004 (1990), pp. 195-198): «Fait remarquable, le rôle de Marey dans de tels débats est régulièrement passé sous silence, bien qu'une grande partie du travail de Bergson sur le temps soit un commentaire de Marey, souvent évoqué même s'il n'est que rarement cité sous son nom» (id., p.195-196).

8 L'appareil exposé porte le numéro 12 (Marey, s.d.[1900]).

9 Bergson fait de la mesure l'apanage de la science moderne. 
à son stade chronophotographique subissent une mutation. Le «cinéma» de Bergson n'est pas celui de Marey, même si les deux relèvent de la chronophotographie. Il s'agira donc ici de comprendre l'évaluation à travers les effets qu'elle produit sur les «objets» auxquels elle s'applique, précisément les concepts du « cinéma».

\section{LE COUPLE CONCEPTUEL DU «CINÉMA» ET DE LA SCIENCE SELON BERGSON}

Bergson nous entraîne dans une analyse détaillée du cinématographe. Comment procède celui-ci pour filmer le défilé d'un régiment, demande Bergson dans L'évolution créatrice? Sa méthode est «de prendre sur le régiment qui passe une série d'instantanés, et de projeter ces instantanés sur l'écran, de manière qu'ils se remplacent très vite les uns les autres ${ }^{10}$. Résumons ce passage célèbre: le cinématographe morcelle le mouvement du régiment en une série de photographies instantanées, les photogrammes, qu'il juxtapose sur la pellicule. Le mouvement reconstitué n'est que le résultat d'une décomposition préalable du mouvement du mobile. L'instantané photographique, tel qu'on le trouve sur la bande du cinématographe, porte donc la charge négative du modèle cinématographique. Sous la plume de Bergson, plusieurs expressions renvoient à ces immobilités extraites du mouvement: on trouve «coupes instantanées ${ }^{11}$, «vues prises ${ }^{12}$, «vues stables» ${ }^{13}$, qui font référence à la photographie et que Bergson emploie au pluriel.

L'analyse du cinématographe le montre, toute la réflexion de Bergson est construite sur l'opposition entre, d'une part, la continuité du mouvement du mobile associé à la durée et, d'autre part, l'immobilité des coupes, des ces photographies, qui sont en fait des photogrammes.

Ce qui intéresse Bergson, ce n'est pas d'opposer l'image photographique à une image en mouvement, projetée sur l'écran - qui n'est en somme qu'un faux mouvement -, mais de montrer que la série d'instantanés fige le mouvement en le décomposant. On le comprend mieux si on replace dans son contexte de démonstration la référence au cinématographe. Très vite, Bergson en vient au commentaire des paradoxes de Zénon, et notamment à celui de la flèche. Puisqu'une flèche en mouvement occupe successivement au minimum deux instants, en chaque seul instant, elle est immobile. Et comme la trajectoire de la flèche peut être décomposée en une série de points - en une série d'instants où la flèche se trouve arrêtée - on peut considérer que, pendant qu'elle se meut, la flèche est immobile tout le long de sont trajet ${ }^{14}$. Les photogrammes sont des équivalents de ces arrêts. Les intervalles quant à eux séparent les points sur la trajectoire du mobile comme les

\footnotetext{
10 L'évolution créatrice (EC), p. 304.

11 EC, p. 250.

12 EC, pp. 177-178, 201, 249.

13 EC, p. 301.

14 EC, p. 308.
} 
photogrammes entre eux sur la pellicule. Ils sont décomposables à l'infini par la science, contrairement au point géométrique ${ }^{15}$, et peuvent se décrire comme une juxtaposition d'instants, de points ou de vues ${ }^{16}$.

Mais, pour Bergson, le mouvement est indécomposable, il se donne d'un seul coup: l'arrêt dans l'instant est une abstraction théorique ou scientifique; il n'y a que des transitions. Les intervalles sont les lieux du changement que la science ne cesse de manquer à force de s'attacher à des vues photographiques, ces instants, ces points géométriques:

Le mouvement glissera dans l'intervalle, parce que toute tentative pour reconstituer le changement avec des états implique cette proposition absurde que le mouvement est fait d'immobilités ${ }^{17}$.

Le commentaire de la flèche de Zénon propose un premier complément au modèle cinématographique en faisant encore une fois de l'instant un arrêt, mais surtout en introduisant le terme d'intervalle, qui peut être articulé au point géométrique, comme au photogramme. Se dégage ainsi un double paradigme, photographie/intervalle, dont les termes sont en opposition:

Tableau I

Couple conceptuel du «cinéma» dans le modèle bergsonien du cinématographe et double paradigme

\begin{tabular}{|c|c|}
\hline Photographie (photogramme) & l'intervalle \\
\hline Instant & Durée \\
\hline Arrêt & Mouvement \\
\hline Point géométrique & Trajectoire \\
\hline
\end{tabular}

Ce tableau ne rend cependant pas exactement compte de l'argumentation bergsonienne. Si l'on peut formuler ainsi la relation entre photographie et intervalle, il convient pourtant d'expliciter le processus analytique qui y conduit, et qui suppose une double définition de l'intervalle. Les termes de durée et de mouvement font l'objet d'une reformulation sous la plume de Bergson, parce qu'ils sont au cœur de la critique portée contre la méthode scientifique. En effet, soit l'inter-

15 Le principe du point géométrique est justement son indivisibilité.

16 Ainsi s'exprime le processus de décomposition de l'intervalle dans la méthode cinématographique: «Sur la continuité de mon devenir j'ai pris une série de vues que j'ai reliées entre elles par le «devenir» en général. Mais il est entendu que je ne puis en rester là. Ce qui n'est pas déterminable n'est pas représentable. (...) Comme la lettre $x$ désigne une certaine inconnue, quelle qu'elle soit, ainsi mon « devenir en général », toujours le même, symbolise ici une certaine transition sur laquelle j'ai pris des instantanés: de cette transition même il ne m'apprend rien. Je vais donc me concentrer tout entier sur la transition et, entre deux instantanés, chercher ce qui se passe. Mais, puisque j'applique la même méthode, j'arrive au même résultat; une troisième vue va simplement s'intercaler entre les deux autres. Indéfiniment je recommencerai, et indéfiniment je juxtaposerai des vues à des vues, sans obtenir autre chose. L'application de la méthode cinématographique aboutira donc ici à un perpétuel recommencement (...)» (EC, pp. 306-307).

17 EC, p. 307 (nous soulignons). Ou encore: «C'est qu'il y plus dans la transition que la série des états, c'est-à-dire des coupes possibles, plus dans le mouvement que la série des positions, c'est-à-dire des arrêts possibles» (EC, p. 313). 
valle est décrit par Bergson du point de vue de la science comme une juxtaposition d'instants; soit il est présenté du point de vue bergsonien comme une transition, une durée, un mouvement pris dans le changement. La «durée» de la science n'est pas la même que celle de Bergson.

Tableau II

\begin{tabular}{|c|c|}
\hline \multicolumn{2}{|c|}{ Intervalle } \\
\hline Point de vue de la science selon Bergson & Point de vue de Bergson \\
\hline Juxtaposition d'instants & Durée, changement, Transition \\
\hline Juxtaposition d'arrêts & Mouvement \\
\hline Trajectoire décomposable & Trajectoire indécomposable $^{18}$ \\
\hline
\end{tabular}

C'est au prix de la redéfinition de la durée que l'intervalle peut être valorisé et associé au temps bergsonien. L'intervalle trouve donc une formulation «positive» au nom de la réalité du mouvement et du temps que Bergson impose à partir de ses propres concepts. Il en va autrement du photogramme et de son paradigme, qui supporte le poids négatif de l'analyse: il reste, dans le discours de Bergson, une pure construction de la science sans rapport avec la réalité changeante. Après l'analyse bergsonienne, les deux éléments constitutifs du couple conceptuel photogramme/intervalle ne sont plus tout à fait au même niveau: ni pour ce qui concerne leur valeur, ni pour ce qui concerne les notions qui les déterminent.

C'est bien par la logique et la force de l'argumentation que les concepts de temps et de mouvement dans leur redéfinition bergsonienne en viennent à qualifier l'un des termes du double paradigme propre au modèle cinématographique: l'intervalle semble alors acquérir une sorte d'autonomie à l'égard de la vue photographique qui reste, elle, rivée à la formulation scientifique. Le résultat s'impose: le couple photographie/intervalle, dont les termes appartiennent à deux sphères de pensée qui s'opposent radicalement, vont être pensés en termes d'opposition.

Le couple photographie/intervalle nous intéresse tout particulièrement parce qu'il définit un paradigme associé au cinématographe, et qu' il se trouve au cœur de l'évaluation bergsonienne de la science. A travers ce couple, les concepts de la science sont directement liés par Bergson à ceux du cinéma. Il s'agira donc de spécifier au maximum ce double paradigme. Quel «cinéma» se trouve derrière la référence bergsonienne et quelle appropriation Bergson opère-t-il de ses concepts? Cela reviendra à préciser le lien qui s'établit entre arrêt et mouvement, instant et durée, photographie et intervalle dans le «cinéma» autour de 1900. Ce n'est qu'ensuite que nous pourrons mesurer l'appropriation bergsonienne à l'œuvre dans son évaluation de la science.

18 On peut parler de trajectoire indécomposable dans le sens où elle renvoie à une action unique. Bergson propose deux manières de parler de la trajectoire: «Au fond, l'illusion vient de ce que le mouvement, une fois effectué, a déposé le long de son trajet une trajectoire immobile sur laquelle on peut compter autant d'immobilités qu'on voudra. (...) On ne voit pas que la trajectoire se crée tout d'un coup, encore qu'il lui faille pour cela un certain temps, et que si l'on peut diviser à volonté la trajectoire une fois créée, on ne saurait diviser sa création, qui est un acte en progrès et non pas une chose» (EC, p. 309). 


\section{LE COUPLE CONCEPTUEL DU «CINÉMA» AU STADE CHRONOPHOTOGRAPHIQUE (MAREY)}

On l'aura compris, ce n'est pas dans le schéma historiographique de l'invention «originelle» du cinématographe Lumière que nous pourrons comprendre les concepts du cinéma auxquels emprunte Bergson, mais en tenant compte de son contexte d'émergence, qui renvoie à la chronophotographie scientifique. Or, il est une idée du cinéma au moment de son émergence où la question de la mesure est essentielle: c'est celle que retient Bergson.

Nous l'avons dit, tout le travail de Marey comme physiologiste s'inscrit dans une finalité: la connaissance du mouvement. Après avoir travaillé avec la méthode graphique, qui permet d'étudier des mouvements invisibles, comme les battements du cœur par exemple, il s'intéresse dès les années 1880 à la locomotion humaine et animale, tout particulièrement, au vol des oiseaux. C'est dans cette nouvelle visée qu'il met au point des instruments fondés sur la technique de l'instantané photographique et destinés à la décomposition du mouvement des mobiles par une série de vues prises à intervalle régulier au moyen d'un appareil à un seul objectif. Marey inventera et réalisera des appareils chronophotographiques pour appliquer ce qu'il définit comme deux méthodes de la chronophotographie: la «méthode sur plaque fixe» et la «méthode sur plaque mobile ${ }^{19}$. Le principe de base est le même: l'alternance entre le photogramme qui capte l'instant d'un mouvement, et l'intervalle où se poursuit son déplacement. Marey travaille aussi à la synthèse du mouvement à partir des vues prises sur les corps en déplacement ${ }^{20}$. La décomposition analytique reste cependant le moment premier de la méthode.

Pour connaître le mouvement, Marey améliore sans cesse ses instruments permettant de capter ce qui échappe à l'œil $n^{21}$. Il observe en physiologiste la position des corps dans leurs déplacements, celle de certains membres par rapport à d'autres, comme aussi le fonctionnement des muscles - notamment à travers les modelés visibles sur les images. Il étudie de plus les phases des mouvements, qui sont périodiques: le battement des ailes, le galop, la marche etc. Mais surtout, il opère différentes mesures pour connaître la vitesse, la force, le travail. C'est parce

19 La distinction est ainsi nommée dans Le Mouvement (Marey, 1894, ch. IV et VII). La chronophotographie sur plaque mobile utilise principalement la pellicule en mouvement, qui s'arrête de manière intermittente devant l'objectif. Le chronophotographe sur plaque fixe opère sur champ obscur et le mobile est enregistré plusieurs fois sur la même plaque photographique, son déplacement assurant les intervalles entre les images.

20 On souligne souvent le désintérêt de Marey pour le cinématographe comme spectacle populaire autour de 1900. Cependant, il faut mettre en avant aussi que la reconstitution du mouvement est une étape de sa méthode, étape qui le retient depuis le début (Marey, 1885, p. 16, fin de la note 1 de page p. 12 ), et dont il souligne les avantages pour l'analyse même (Marey, 1890, pp. 181-182, ou Marey, 1894, p. 310). La synthèse du mouvement se fait notamment au ralenti pour permettre l'observation fine.

${ }^{21}$ Marey vante dès Le Développement de la méthode graphique par l'emploi de la photographie l'avantage de la photographie sur l'observation à l'œil nu: «Dans une photographie, tout est représenté, et, sur une image chargée de détails, si nous ne saisissons pas tout dès le premier coup d'œil, nous pouvons revenir plus tard à un nouvel examen de cette image et y découvrir ce qui nous avait échappé tout d'abord». La photographie est «une mémoire fidèle » (Marey, 1885, p. 3). 
que la mesure et les calculs font partie de la visée du scientifique que les instruments élaborés pour les expériences doivent garantir la possibilité comme la justesse des mesures sur les images mêmes. La «spécialité» de Marey dès la méthode graphique est de fonder son travail sur la recherche de capteurs capables de transcrire directement le mouvement physiologique: le mouvement en quelque sorte s'écrit lui-même ${ }^{22}$. La mesure est donc déterminée par les instruments que Marey fabrique en innovant. Avec la chronophotographie, la justesse de la transcription est tout aussi essentielle. Elle est garantie par la rapidité de la trace lumineuse et l'instantanéité de la réaction chimique propre à la photographie instantanée: l'inertie est minimale dans la transmission de l'information au moment de l'enregistrement.

Cependant, si la méthode chronophotographique de Marey permet l'expérience scientifique, les mesures, et les calculs, c'est fondamentalement parce qu'elle impose des intervalles réguliers entre les photographies: la fréquence de prise de vue est fixée en fonction de chaque expérience. L'intervalle régulier entre les instantanés photographiques est définitoire de la chronophotographie chez Marey: il est une condition de possibilité de l'expérience scientifique avant d'être celle du cinéma ${ }^{23}$. La maîtrise de la variable temporelle permet en effet d'opérer des mesures sur les images photographiques pour calculer, par exemple, la distance parcourue par unité de temps. Chaque photogramme traduit la place que le mobile occupe dans l'espace; chaque point d'une figure sur l'image renvoie à ce même point sur le mobile photographié. Et comme l'intervalle se comprend à la fois comme une distance, celle qui sépare deux photogrammes entre eux, et comme une durée, celle qui mesure le temps parcouru entre deux prises photographiques, la variation de longueur mesurée entre les photogrammes indique donc la variation de vitesse du mobile photographié. On peut alors constater sur les images mêmes l'accélération ou le ralentissement du mobile (voir Illustration).

Comprendre le rapport entre intervalle et photogramme dans la série chronophotographique, c'est comprendre les mesures opérées par Marey ${ }^{24}$.

1. Lorsque Marey mesure la vitesse, il s'occupe d'un seul mobile se déplaçant devant un objectif. Il lui faut rapporter la distance parcourue par le mobile au temps pris pour la parcourir. La mesure est par définition classique: dans un premier temps, on rapporte une donnée à un étalon fixé par convention, soit lorsqu'on mesure la distance entre les intervalles (le mètre sur le photogramme) soit lorsqu'on mesure le temps qui sépare les poses. Cette dernière

22 «Le «tracé» doit (...) être tenu pour l'expression même de la nature, sans écran, sans écho, sans interférence: il est fidèle, clair et surtout universel. Il ne reste qu'à traduire ce que la nature nous communique elle-même sur elle-même» (Dagognet, 1987, p. 48). François Dagonet fait de la recherche sur les capteurs un des aspects fondamentaux du scientifique Marey, (Dagognet, 1987, notamment pp. 45-49). Voir aussi Michel Frizot dans sa réflexion sur le «point zéro» (Frizot, 2001, pp. 101-103).

23 Elle est nécessaire aussi à la synthèse du mouvement. C'est un des points qui distingue les travaux de Marey des photographies de Muybridge sur le galop du cheval.

${ }^{24}$ Parce qu' «une mesure telle quelle n'a pas de sens et qu'il faut toujours, pour éviter le terrorisme du chiffrage brut, réinsérer le résultat à l'intérieur de la méthode qui l'a produit» («Qu'est-ce que mesurer? Peut-on tout mesurer?», Dagognet, 1999, p. 47). Voir aussi l'article de François Dagognet «Mesure » dans le Dictionnaire d' histoire et de philosophie de sciences (Lecourt, 1999). 
est une mesure de contrôle, puisque le temps est fixé pour chaque expérience. C'est avec ces deux données qu'on peut calculer la vitesse. Sur les photographies apparaissent les instruments de mesure: le mètre et le chronographe.

2. Mais les expériences de Marey imposent une autre forme de mesure qui relève d'une autre définition, tout aussi classique d'ailleurs. En fixant la variable temporelle de la prise photographique, c'est-à-dire la fréquence, Marey impose la base temporelle fondamentale par rapport à laquelle pourront être mesurés soit le mouvement uniforme du mobile, soit ses variations de vitesse. Le rapport entre vitesse du mobile et intervalle régulier peut être comparé à celui qui lie, en musique, le rythme des notes au battement régulier de la mesure (cadence); ou, en versification, à celui qui permet à la syntaxe du poème de se déployer dans l'espace réglé du mètre. En somme, la mesure désigne la succession de divisions temporelles d'égale valeur. La mesure métrique de l'intervalle est la condition de possibilité de l'expérience scientifique de Marey, et permet toutes les déterminations de grandeurs par comparaison avec un étalon (déf. 1).

La mesure détermine pour trois raisons au moins le couple photographie/intervalle dans l'expérience chronophotographique.

1. Parce qu'elle est une finalité de l'expérience: Marey veut calculer la vitesse du mobile à partir des mesures opérées (déf. 1).

2. Parce que la mesure entendue comme fréquence (déf. 2) est la condition de possibilité de l'expérience: elle est nécessaire au calcul de la vitesse. Or, comme la durée de l'intervalle est déterminée par la fréquence de la prise photographique, on peut dire que l'intervalle et le photogramme sont interdépendants: l'un se définit en référence à l'autre. La relation entre les deux termes ne relève pas de l'opposition, mais de la corrélation. De plus, leur interdépendance est fonction de la définition de la chronophotographie qui impose physiquement leur alternance temporelle et visuelle. Ainsi est construit le couple conceptuel fondamental de la chronophotographie.

3. La mesure est en troisième lieu un moment nécessaire dans la mise en place de l'un et l'autre paramètres. En effet, si l'on peut opérer des mesures sur les photographies, c'est que le fonctionnement des appareils est lui-même régi et contrôlé par des mesures. Ainsi, la vitesse de rotation du disque obturateur doit être connue et donc contrôlée par une mesure ${ }^{25}$. Avec le nombre d'ouvertures, elle détermine la fréquence des prises de vue, c'est-à-dire, finalement, la durée des intervalles. Est mesurée aussi l'ouverture de l'obturateur, qui doit être choisie pour garantir la netteté de l'image, ce qui relève de la technique de l'instantané ${ }^{26}$. L'intervalle comme le photogramme sont le produit de diffé-

25 Dans Le vol des oiseaux par exemple, Marey explique comment « régler exactement les durées de ces intervalles» (Marey, 1890, p. 142).

26 «(...) d'après l'espace angulaire que représentent les fenêtres, on saura la durée du temps de pose» (Marey, 1890, p.142). La photographie instantanée suppose la mesure de l'ouverture de l'obturateur mais aussi la mesure de la rapidité de la réaction chimique imposée par le support. 
rentes mesures, qui concernent spécifiquement chacun des deux éléments du couple conceptuel de la chronophotographie. Photographie et intervalle sont pensés, au moment de leur production, de leur utilisation ou de leur théorisation, comme des durées mesurables, et par conséquent décomposables en unités de temps. Si pour Marey les photographies renvoient bien à des instants du mouvement du mobile, ces instants sont bien mesurables et décomposables $^{27}$. Ils ne peuvent être décrits uniquement comme des points géométriques à l'instar de l'argumentation bergsonienne ${ }^{28}$. Le couple photographie/intervalle, que Marey lui-même renvoie à instant/intervalle, ce couple est pensé de manière homogène $e^{29}$.

Tableau III

Couple conceptuel du «cinéma» au stade chronophotographique et double paradigme Modèle mareysien

\begin{tabular}{|c|c|}
\hline Photographie (photogramme) & Intervalle (distance) \\
\hline Instant $^{30}$ & Intervalle (durée) \\
\hline Durée & Durée \\
\hline Mouvement $($ du mobile) & 31 \\
\hline
\end{tabular}

Force est de constater que le couple de la chronophotographie se distingue de celui qui se dégage du modèle cinématographique proposé par Bergson. Dans le contexte bergsonien qui fait du mouvement l'essence de la réalité, introduire l'arrêt pour l'opposer au mouvement, et l'instant pour l'opposer à la durée, c'est

27 C'est un point que Marey souligne dans La méthode graphique: «(...) la chronographie est admirable; véritable microscope du temps, elle montre que l'instant indivisible dont on parle souvent n'existe pas, et que parfois des actes réguliers, rythmés et coordonnés d'une manière parfaite tiennent dans un centième de seconde» (Marey, 1878, p.XII). Voir Michel Frizot, (2001, pp. 103-107).

28 Bergson associe l'instant de la science à sa stricte traduction graphique et géométrique: le point. Mais l'expérience scientifique de la chronophotographie pense et construit l'instant comme une durée, bien que sa notation graphique puisse être un point. Pour être comprise, la notation qui permet la mesure doit être réintroduite dans son contexte de production.

29 Le terme est employé ici sans référence à l'usage important qu'en fait Bergson dans la critique du temps de la science, le temps homogène. Nous l'utiliserons, de même que le terme «hétérogène », pour qualifier la relation entre les concepts qui nous intéressent, compte tenu des notions qui les définissent.

${ }^{30}$ Cet instant-durée peut être construit épistémologiquement de manière bien plus précise. Voir «L'instantané cinématographique: relire Etienne-Jules Marey (Tortajada, 2008, à paraître).

31 Nous n'avons pas développé ici la question du mouvement. Cependant, il en va du mouvement comme de la mesure: il conditionne les deux paramètres. Dans la fabrication du photogramme comme de l'intervalle, l'expérience impose de prendre en considération le mouvement du mobile. Le concept de mouvement est impliqué dans la notion d'intervalle, car pour calculer ce dernier, il faut tenir compte de la vitesse de déplacement des corps afin d'éviter la superposition des images. De même, le concept de mouvement est impliqué dans la notion d'instant photographique, car le temps de pose est défini en fonction du mouvement du mobile pour parvenir à une image nette sans bougés. 
articuler ensemble des données de nature différente, ontologiquement ${ }^{32}$ différentes, à l'intérieur du couple conceptuel photographie/intervalle: durée et intervalle sont en effet redéfinis à partir des catégories de Bergson. Le mouvement et la durée sont l'essence même de la vie et de la matière. L'arrêt est au contraire une abstraction de la science ou du langage, et l'instant un découpage théorique. C'est dire que Bergson fait fonctionner ensemble deux notions, photogramme et intervalle, qui articulent chacune, au moment de leur reformulation, des concepts hétérogènes, et dont la relation ne se définit pas par l'alternance, mais par l'opposition. Cette hétérogénéité, que Bergson tient à mettre en évidence parce qu'il valorise avant tout sa définition de la réalité et le mouvement du mobile, fonde justement sa critique de la science. C'est le premier déplacement imposé par la réévaluation bergsonienne aux concepts du cinéma au moment Marey, qui sont aussi de fait ceux de la chronophotographie.

Tableau IV

\begin{tabular}{|c|c|c|}
\hline Rapport Photo/intervalle & Définitions des termes & Relation \\
\hline Marey & Homogènes & Alternance, corrélation \\
\hline Bergson & Hétérogènes & Opposition \\
\hline
\end{tabular}

\section{LA VALEUR DE L'INSTANT}

La réappropriation conceptuelle engagée par Bergson modifie sous d'autres aspects encore l'idée du cinéma à laquelle il fait référence. Nous n'en traiterons qu'un ici, à titre d'exemple, parce qu'il concerne le couple définitoire de la chronophotographie.

C'est dans la différenciation entre science moderne et antique que se joue un moment essentiel pour la compréhension du modèle cinématographique: la théorisation par Bergson de l'instant quelconque. Celui-ci, nous le verrons, est pensé à partir de l'intervalle régulier de la chronophotographie. Pourtant, la distinction que Bergson introduit à cette occasion entre les deux sciences transforme paradoxalement l'idée même du «cinéma» à laquelle il fait référence.

Il faut pour cela en venir à un troisième temps de la démonstration que développe Bergson autour du cinématographe dans le chapitre IV de L'évolution créatrice. Bergson reprend encore une fois le même argument: les deux sciences, écrit-il, ne s'intéressent qu'à des immobilités, et c'est en cela que le fonctionnement du cinématographe les résume ou les modélise. Ce qui caractérise la science antique, comme sa philosophie, c'est qu'elle «s'installe dans l'immuable ${ }^{33}$, qu'elle valorise au dépend du mouvement; elle travaille avec des Idées et des Formes, qui sont des immobilités. Décrites du point de vue bergsonien, les

32 Sur la dimension ontologique de Bergson, et plus particulièrement sur le lien que tisse le philosophe entre substance et apparence, voir Henri Hude (1990, pp. 138-139). Voir aussi Gilles Deleuze (1968, p. 58 et suiv.) autour de Matière et mémoire.

33 EC, p. 315. 
Formes, que l'esprit isole et emmagasine dans des concepts, ne sont alors que des vues prises sur la réalité changeante. Elles sont des moments cueillis le long de la durée, et, précisément parce qu'on a coupé le fil qui les reliait au temps, elles ne durent plus. (...) Elles entrent dans l'éternité si l'on veut; mais ce qu'elles ont d'éternel ne fait plus qu'un avec ce qu'elles ont d'irréel ${ }^{34}$.

C'est la critique essentielle de Bergson contre la science antique. Comme les vues instantanées, les Formes sont des instants extraits de la durée, pensées comme des arrêts: leur nature de signe le leur impose ${ }^{35}$. La science moderne, comme la science antique, procède selon la méthode cinématographique, car comme elle, elle appréhende la réalité à travers des signes ${ }^{36}$. On revient encore au même critère, celui de l'arrêt du mouvement pour spécifier l'instant.

Ce qui distingue la science moderne, c'est qu'elle s'intéresse à la nature de l'instant: «(...) la science antique croit connaître suffisamment son objet quand elle en a noté des moments privilégiés, au lieu que la science moderne le considère à n'importe quel moment.» ${ }^{37}$

Bergson prend pour exemple le calcul de la pesanteur. Pour Galilée, il n'y a pas de moment essentiel, pas d'instant privilégié. Etudier le corps qui tombe, c'est le considérer à n'importe quel moment de sa course: "La vraie science de la pesanteur sera celle qui déterminera, pour un instant quelconque du temps, la position du corps dans l'espace.» «Tous les instants se valent ${ }^{38}$, parce qu'ils renvoient à une série de points sans sélection préalable, comme dans le cinématographe les photogrammes, qui ne sont déterminés que par la fréquence de prise de vue sans tenir compte de la prégnance de tel ou tel élément de la réalité. Dans ce sens, la théorisation de l'intervalle régulier dans la chronophotographie s'accorde avec la notion d'instant quelconque.

Bergson précise le sens de l'instant quelconque en l'opposant à l'instant prégnant, notion empruntée au domaine de l'esthétique:

Du galop d'un cheval notre œil perçoit surtout une attitude caractéristique, essentielle ou plutôt schématique, une forme qui paraît rayonner sur toute une période et remplir ainsi un temps de galop: c'est cette attitude que la sculpture a fixée sur les frises du Parthénon. Mais la photographie instantanée isole n'importe quel moment; elle les met tous au même rang, et c'est ainsi que le galop d'un cheval s'éparpille pour elle en un nombre aussi grand qu'on voudra d'attitudes successives, au lieu de se ramasser en une attitude unique, qui brillerait en un instant privilégié et éclairerait toute une période ${ }^{39}$.

34 EC, pp. 316-317.

35 «(...) la condition générale du signe, qui est de noter sous forme arrêtée un aspect fixe de la réalité» (EC, p. 328).

36 EC, p. 328.

37 EC, p.330. Et encore: «Il y a entre ces deux sciences le même rapport qu'entre la notation des phases d'un mouvement par l'œil et l'enregistrement beaucoup plus complet de ces phases par la photographie instantanée. C'est le même mécanisme cinématographique dans les deux cas, mais il atteint, dans le second, une précision qu'il ne peut pas avoir dans le premier» (EC, p.331).

38 EC, pp. 330-331.

39 EC, p. 332. 
Le commentaire renvoie à la définition développée et discutée au XVIII ${ }^{\mathrm{e}}$ siècle de l'instant prégnant, à laquelle Laocoon de Lessing donne une acception célèbre: il est cet instant unique proposant au spectateur une synthèse temporelle, le propre des arts de l'espace, la peinture au premier chef ${ }^{40}$. L'exemple de la frise du Parthénon s'inscrit parfaitement dans ce contexte de référence ${ }^{41}$.

Si l'instant quelconque peut clairement s'expliquer par l'intervalle régulier du cinématographe, l'instant prégnant est non seulement totalement étranger au fonctionnement photogrammatique de ce dernier, mais bien réellement contradictoire avec lui par définition ${ }^{42}$. Or, la science antique comme la science moderne relèvent selon Bergson de la méthode cinématographique, qui a fonction de modèle.

Cela pose en effet problème ${ }^{43}$. De fait, ce qui distingue justement le « cinéma», ce qui est fondamental dans le dispositif chronophotographique, c'est l'intervalle régulier. Que Bergson puisse passer outre cette condition pour associer le modèle cinématographique au domaine de la science antique, où règne l'instant prégnant, montre que, pour lui, cet aspect est second par rapport à la notion d'arrêt $t^{44}$ : l'essentiel est que la photographie instantanée fige le mouvement du mobile, ce qui réunit les deux sciences. En faisant de l'immobilité une priorité contre l'intervalle régulier, Bergson minimise l'élément déterminant de la chronophotographie comme de la réalité historique du cinématographe, qui veut que l'intervalle régulier soit une condition nécessaire de sa définition.

Il le minimise, et aussi le détourne, car il transforme la relation entre instant et intervalle. En effet, l'intervalle se comprend, dans le contexte de la chronophotographie, comme la durée - ou la distance - qui sépare les vues entre elles dans une série de photogrammes. L'intervalle régulier est un élément explicite et définitoire de l'instant quelconque. Il en va tout autrement pour l'instant prégnant: la notion d'intervalle non seulement ne le définit en rien mais en plus pose problème. Pour

40 «Si l'artiste ne peut choisir qu'un seul instant de la nature toujours changeante; si, en outre, la peinture ne peut utiliser qu'un unique point de vue pour saisir cet unique instant; si, d'autre part, ses œuvres sont faites pour être non seulement vues, mais contemplées longuement et souvent, il est alors certain que cet instant et ce point de vue uniques ne sauraient être choisis trop féconds. Or cela seul est fécond qui laisse le champ libre à l'imagination (...) Or dans le cours d'une passion, l'instant de paroxysme est celui qui jouit le moins de ce privilège (...) Puisque cet instant acquiert par l'art une durée immuable, il ne doit pas exprimer ce qui ne se conçoit que comme transitoire.» (Lessing, 1990 (1766), p. 55-56) et encore «Pour ses compositions, qui supposent la simultanéité, la peinture ne peut exploiter qu'un seul instant de l'action et doit par conséquent choisir le plus fécond, celui qui fera le mieux comprendre l'instant qui précède et celui qui suit.» (id. p.120)

${ }^{41}$ Comme elle renvoie aussi aux débats sur la chronophotographie qui se sont développés autour de la question du galop du cheval et de sa représentation picturale, débats liés au contexte de rencontre entre Muybridge et Marey. Sur Marey et Muybridge, voir Marta Braun (1992).

42 Il y a certes des instants prégnants au cinéma, mais ils se situent au niveau du plan, et non pas du photogramme.

43 «La méthode cinématographique s'impose donc à notre science, comme elle s'imposait déjà à celle des anciens » (EC, p.329). Gilles Deleuze s'attarde à ce moment particulier de l'argumentation de Bergson pour y relever une «hésitation», qu'il résoudra en réintroduisant sa propre thèse, et fonder l'image-mouvement, image du cinéma, dans le bergsonisme (Deleuze, 1983, pp. 16-17). Nous suivons ici une autre piste.

44 «La différence est profonde. Elle est même radicale par un certain côté. Mais du point de vue où nous l'envisageons, c'est une différence de degré plutôt que de nature» (EC, p. 331). 
qu'il y ait intervalle, il faut au moins deux instants. La notion d'intervalle fonctionne donc bien avec celle de série, qui suggère la pluralité des instants et donc l'existence même des intervalles qui les séparent. La notion d'instant prégnant, de son côté, s'accorde en effet difficilement à celle de «série», car elle présente le temps comme une synthèse, en un seul instant, du passé et de l'avenir. L'instant prégnant est l'instant unique: il se dégage de tous les autres, et contient l'avant et l'après. Un temps qui se donne en un seul instant peut-il être décrit en termes d'intervalles? L'idée d'instant prégnant résiste au modèle chronophotographique qui impose une pluralité d'instants. Si dans un tel contexte on choisit de maintenir comme pertinente la notion d'intervalle ${ }^{45}$, ce ne peut être qu'au prix de sa redéfinition. En appliquant le modèle cinématographique à la science antique comme il le fait après avoir défini la spécificité de l'instant quelconque, Bergson opère une réappropriation conceptuelle $\mathrm{du}$ couple instant/intervalle, définitoire $\mathrm{du}$ «cinéma» au stade chronophotographique.

\section{CONCLUSION}

On peut résumer ici les étapes de l'évaluation bergsonienne de la science dans L'évolution créatrice:

1. Bergson décrit l'objet évalué, la science, en isolant les concepts pertinents: il retient le temps, l'instant, le mouvement, l'intervalle et la mesure.

2. Il éclaire et explique ces concepts en les plaçant dans un cadre qu'il définit comme le «modèle cinématographique». Pour cela, il s'impose une description précise du dispositif et de la machine du «cinématographe», et dégage de son fonctionnement les concepts «cinématographiques» qu'il applique à la description de la science. Nous avons pu constater que ces concepts s'inscrivent dans le contexte de la chronophotographie. Jusqu'à un certain point, le «cinématographe» de Bergson est un chronophotographe tel que Marey le construit et le théorise dans sa pratique scientifique: les concepts de temps, de mouvement, d'intervalle, d'instant et de mesure sont bien aussi les siens.

3. Or, ces concepts qui apparaissent dans les deux discours - celui de Bergson et celui de Marey - sont en fait différents lorsqu'on les explique par leur construction épistémologique. Les concepts de la chronophotographie acquièrent une nouvelle valeur lorsqu'ils entrent dans le système de cohérence bergsonien. Il ne suffit pas de s'en tenir à la liste des concepts; il faut voir comment les uns et les autres entrent en relation dans leur contexte d'utilisation. Ce qui fonde la chronophotographie, c'est le rapport entre photographie et intervalle, couple conceptuel fondamental auquel sont associés deux paradigmes: ce rapport est constitutif de l'expérience scientifique comme du fonctionnement cinématographique.

45 C'est nous semble-t-il ce que fait Bergson implicitement et paradoxalement lorsqu'il décrit les moments de la science antique par «les moments cueillis le long de la durée» cité plus haut. 
Mais le lien qui fait tenir ensemble ce couple conceptuel est fondamentalement différent chez Bergson et Marey. Ce qui, dans la chronophotographie est appréhendé comme une alternance et une corrélation, devient chez Bergson une opposition: cette opposition est fondamentale et irréductible car elle articule des éléments hétérogènes. Pour Marey, au contraire, les vues et les intervalles, déterminés tous deux par la mesure, sont définis de manière homogène.

Mais la réappropriation bergsonienne va plus loin encore dans l'utilisation du cinématographe. Bergson se détourne du couple essentiel de la chronophotographie pour en privilégier un autre: l'arrêt opposé au mouvement du mobile. L'intervalle régulier n'est pas une notion déterminante du modèle. Mieux encore, il devient une notion incertaine. L'intervalle de la chronophotographie semble être le grand perdant conceptuel de cette reformulation bergsonienne: soit qu'il se trouve minimisé à l'intérieur du modèle bergsonien, soit qu'il devient difficilement saisissable, contraint à l'aporie qu'on lui impose de s'articuler à l'instant prégnant.

Cette transformation opérée dans la réévaluation de la science par Bergson marquera l'histoire du cinéma qui ne cessera de se référer à lui de manière explicite ou implicite. Nous ferons l'hypothèse que la reformulation bergsonienne du double paradigme cinématographique deviendra la référence pour une grande part de la théorie du cinéma au $\mathrm{XX}^{\mathrm{e}}$ siècle.

\section{Université de Lausanne}

\section{BIBLIOGRAPHIE}

Agambem, Giorgio, Qu' est-ce qu'un dispositif?, Paris, Payot,Rivages, 2007 (2006).

Albera, François, Tortajada, Maria, «L'Epistémè «1900» Le cinématographe, nouvelle technologie du XX $X^{e}$ siècle/The Cinema, A New Technology for the 20th Century, Lausanne, Payot, 2004, pp. 45-62.

Albera, François, «Pour une épistémographie du montage. Le moment-Marey», in F. Albera, Braun, Marta, Gaudreault, André (dir.), Arrêt sur image, fragmentation du temps. Aux sources de la culture visuelle moderne / Stop-motion, Fragmentation of Time, Exploring the Roots of Modern Visual Culture, Lausanne, Payot, 2002, pp.31-46.

Bachelard, Gaston, La formation de l' esprit scientifique, Paris, Vrin, 1999 (1938).

Bazin, André, Qu' est-ce que le cinéma? Paris, Cerf, 1985 (1975).

Bergson, Henri, Matière et mémoire, Paris, PUF (Quadrige), 1997 (1896).

Bergson, Henri, L'évolution créatrice, Paris, PUF (Quadrige), 1998 (1907).

Château, Dominique, Cinéma et philosophie, Paris, Nathan (Cinéma), 2003.

Dagognet, François, Etienne-Jules Marey. La passion de la trace, Paris, Hazan, 1987.

Dagognet, François, Les outils de la réflexion, Le Plessis-Robinson, Institut Synthélabo, 1999.

Braun, Marta, Picturing Time, The Work of Etienne-Jules Marey, Chicago/London, University of Chicago Press, 1992.

Deleuze, Gilles, Le bergsonisme, Paris, PUF, 1968.

Deleuze, Gilles L'image-mouvement, Paris, Minuit, 1983.

Deleuze, Gilles, L'image-temps, Paris, Minuit, 1985.

Epstein, Jean, L'intelligence d' une machine, Paris, Jacques Melot, 1946.

Frizot, Michel, Etienne-Jules Marey chronophotographe, Paris, Nathan/Delpire, 2001. 
Frizot, Michel, «Les courbes du temps. L'image graphique et la sensation temporelle», Pascal Russo (dir), Aux origines de l'abstraction (Catalogue), Paris, Musée d'Orsay, février 2004, pp. 69-83.

Hude, Henri, Bergson II, [Paris], Editions universitaires, 1990.

Lecourt, Dominique (dir), Dictionnaire d'histoire et de philosophie de sciences, Paris, PUF, 1999. Lessing, Locoon, Paris, Hermann, 1990 (1766).

Mannoni, Laurent, Etienne-Jules Marey. La mémoire de l' œil, Milna/Paris, Mazzotta/La Cinémathèque française, 1999.

Marey, Etienne-Jules, La méthode graphique dans les sciences expérimentales et particulièrement en physiologie et en médecine, Paris, Masson, 1878.

Marey, Etienne-Jules, Développement de la méthode graphique par l'emploi de la photographie. Supplément à La Méthode graphique dans les sciences expérimentales, Paris, Masson, 1885.

Marey, Etienne-Jules, Le vol des oiseaux, Paris, Masson, 1890.

Marey, Etienne-Jules, Le mouvement, Nîmes, Jacqueline Chambon, 2002 (1894).

Marey, Etienne-Jules, «Exposition d'instruments et d'images relatifs à l'histoire de la chronophotographie», par le Docteur Marey, membre de l'Institut, Musée centennal de la classe 12 (photographie) à l' Exposition universelle internationale de 1900 à Paris, Métrophotographie et chronophotographie, Saint-Cloud, impr. Belin, s.d

Rabinbach, Anson, Le moteur humain. L'énergie, la fatigue et les origines de la modernité, Paris, La Fabrique, 2004 (1990).

Tortajada, Maria, «Archéologie du cinéma: de l'histoire à l'épistémologie », Cinémas «Histoires croisées des images: Objets et méthodes », (dir. Edouard Arnoldy), vol. 14, nos 2-3, printemps 2004, pp. 19-52.

Tortajada, Maria, «L'instantané cinématographique: relire Etienne-Jules Marey», 2008, à paraître.

Vouilloux, Bernard, «La critique des dispositifs », Critique, Tome LXII, no 718, mars 2007, pp.152168. 